\title{
Towards systems metabolic engineering of streptomycetes for secondary metabolites
} production

Robertsen, Helene Lunde; Weber, Tilmann; Kim, Hyun Uk; Lee, Sang Yup

Published in:

Biotechnology Journal

Link to article, DOI:

10.1002/biot.201700465

Publication date:

2017

Document Version

Peer reviewed version

Link back to DTU Orbit

Citation (APA):

Robertsen, H. L., Weber, T., Kim, H. U., \& Lee, S. Y. (2017). Towards systems metabolic engineering of streptomycetes for secondary metabolites production. Biotechnology Journal, [1700465].

https://doi.org/10.1002/biot.201700465

\section{General rights}

Copyright and moral rights for the publications made accessible in the public portal are retained by the authors and/or other copyright owners and it is a condition of accessing publications that users recognise and abide by the legal requirements associated with these rights.

- Users may download and print one copy of any publication from the public portal for the purpose of private study or research.

- You may not further distribute the material or use it for any profit-making activity or commercial gain

- You may freely distribute the URL identifying the publication in the public portal 


\section{Review}

Towards systems metabolic engineering of streptomycetes for secondary metabolites production $^{\dagger}$

Helene Lunde Robertsen ${ }^{1}$

Tilmann Weber ${ }^{1}$

Hyun Uk Kim²

Sang Yup Lee ${ }^{1,2}$

${ }^{1}$ The Novo Nordisk Foundation Center for Biosustainability, Technical University of Denmark, 2800 Kongens Lyngby, Denmark.

${ }^{2}$ Department of Chemical and Biomolecular Engineering (BK21 Plus Program), Korea Advanced Institute of Science and Technology (KAIST), Yuseong-gu, Daejeon 306-701, Republic of Korea

Correspondence: Prof. Sang Yup Lee, Department of Chemical and Biomolecular Engineering, KAIST, 291 Daehak-ro, Yuseong-gu, Daejeon 34141, Republic of Korea.

E-mail: leesy@kaist.ac.kr

Dr. Tilmann Weber, The Novo Nordisk Foundation Center for Biosustainability, Technical University of Denmark, Kemitorvet 220, 2800 Kongens Lyngby, Denmark.

E-mail: tiwe@biosustain.dtu.dk

${ }^{\dagger}$ This article has been accepted for publication and undergone full peer review but has not been through the copyediting, typesetting, pagination and proofreading process, which may lead to differences between this version and the Version of Record. Please cite this article as doi: [10.1002/biot.201700465].

This article is protected by copyright. All rights reserved

Received: September 12, 2017 / Revised: October 20, 2017 / Accepted: October 25, 2017 


\begin{abstract}
Streptomycetes are known for their inherent ability to produce pharmaceutically relevant secondary metabolites. Discovery of medically useful, yet novel compounds has become a great challenge due to frequent rediscovery of known compounds and a consequent decline in the number of relevant clinical trials in the last decades. A paradigm shift took place when the first whole genome sequences of streptomycetes became available, from which silent or “cryptic” biosynthetic gene clusters (BGCs) were discovered. Cryptic BGCs reveal a so far untapped potential of the microorganisms for the production of novel compounds, which has spurred new efforts in understanding the complex regulation between primary and secondary metabolism. This new trend has been accompanied with development of new computational resources (genome and compound mining tools), generation of various high-quality omics data, establishment of molecular tools and other strain engineering strategies. They all come together to enable systems metabolic engineering of streptomycetes, allowing more systematic and efficient strain development. In this review, we present recent progresses within systems metabolic engineering of streptomycetes for uncovering their hidden potential to produce novel compounds and for the improved production of secondary metabolites.
\end{abstract}

Keywords: Biosynthetic gene clusters - Genome mining - Secondary metabolites Streptomycetes - Systems metabolic engineering 
Abbreviations: antiSMASH, antibiotics and Secondary Metabolites Analysis Shell; ARC, antibiotic-remodeling compound; ARTS, Antibiotics Resistant Target Seeker; BGC, biosynthetic gene cluster; Cas9, CRISPR-associated protein; CDA, calcium-dependent antibiotic; ChIP, Chromatin ImmunoPrecipitation; CRISPR, Clustered Regularly Interspaced Short Palindromic Repeats; dRNA-seq, differential RNA sequencing; EFMA, Elementary Flux Mode Analysis; FBA, flux balance analysis; FSEOF, Flux Scanning based on Enforced Objective Flux; GARLIC, Global Alignment for natuRaL-products chemInformatiCs; GC, gas chromatography; GCF, Gene Cluster Family; GNPS, Global Natural Products Social Molecular Networking; GRAPE, Generalized Retrobiosynthetic Assembly Prediction Engine; GSMM, genome-scale metabolic models; HR, homologous recombination; IMG-ABC, Integrated Microbial Genomes Atlas of Biosynthetic gene Clusters; LC-MS/MS, liquid chromatography tandem mass spectrometry; MFA, metabolic flux analysis; MIBiG, Minimum Information on Biosynthetic Gene Clusters; MOMA, Minimization Of Flux Adjustment; NHEJ, non-homologous end joining; NMR, Nuclear magnetic resonance; NRP, non-ribosomal peptide; PAM, protospacer adjacent motif; PI, pristinamycin I; PII, pristinamycin II; PK, polyketide; PRISM, Prediction Informatics for Secondary Metabolomes; Ribo-seq, ribosome profiling; sgRNA, single guide RNA; SMBP, The Secondary Metabolite Bioinformatics Portal; ssRNA-seq, strand-specific RNA sequencing; TFR, TetR family transcriptional regulator; WGCNA, weighted correlation network analysis 


\section{Introduction}

Streptomycetes are filamentous gram-positive bacteria predominantly found in soil and water environments. The bacteria are recognized for their capabilities to produce secondary metabolites with clinically relevant applications, some of which include antibiotics such as streptomycin and daptomycin, anthelmintic compound avermectin, immunosuppressant tacrolimus (FK-506), and anti-cancer agents bleomycin and doxorubicin (for review, see [1]). Despite the proven clinical effects of secondary metabolites, drug discovery in streptomycetes has witnessed a decline in success rate ever since the golden age of antibiotics experienced from late 1940s to 1960s [2]. A paradigm shift begun in the early 2000s when the complete genome sequences of a model organism Streptomyces coelicolor [3] and an industrial strain Streptomyces avermitilis [4] were published. In the case of S. coelicolor, the $8.6 \mathrm{Mb}$ linear chromosome was found to harbor several cryptic or silent biosynthetic gene clusters (BGCs) in addition to already known BGCs of actinorhodin, undecylprodigiosin and calciumdependent antibiotic (CDA). These silent BGCs showed indications of possible biosynthesis of secondary metabolites, but interestingly, no corresponding compounds were detected under standard laboratory growth conditions. In the following years, many of these silent BGCs were successfully activated and their corresponding secondary metabolites were characterized, including iron-chelators desferrioxamine [5] and coelichelin [6,7], sesquiterpene antibiotic albaflavenone [8], and polyketide (PK) alkaloid coelimycin [9]. The initial successes in identifying novel secondary metabolites from silent BGCs have led to the subsequent increases in the genome sequencing of streptomycetes in the last decade (Fig. 1).

Availability of genome data has spurred advances in relevant technologies covering computational resources (in particular, genome and compound mining tools), high-throughput (omics) techniques as well as molecular tools. Genome mining tools such as antibiotics and Secondary Metabolites Analysis SHell (antiSMASH) [10-12] and Prediction Informatics for Secondary Metabolomes (PRISM) [13,14] have greatly improved our ability to survey the genomic potential of strains, and allowed for initial prioritization of engineering efforts. Also, 
omics techniques such as transcriptomics and proteomics have contributed to understanding the complex regulatory networks employed by streptomycetes to balance primary and secondary metabolism $[15,16]$. Availability of such genome and expression data has further enabled construction of genome-scale metabolic models (GSMMs) of streptomycetes to describe metabolic pathways and predict optimal routes for the production of secondary metabolites [17].

With high-quality whole genome sequences and an array of suitable genome/compound mining and molecular cloning tools readily at hand, the secondary metabolite research is now moving towards systems metabolic engineering of streptomycetes in order to fully harness their potential to produce medically valuable secondary metabolites [18]. Systems metabolic engineering brings in recent developments experienced in the fields of systems and synthetic biology, and provides a more systematic and efficient approaches for strain engineering [19]. However, streptomycetes and other secondary metabolite-producing microbes require additional sophisticated analyses concerned with secondary metabolite BGCs before strains are engineered at the systems level for the novel compound production and the improved production titers [17]. To this end, here we review recent progress within the field of systems metabolic engineering of streptomycetes with focuses on the additional sophisticated analyses unique to streptomycetes as well as recent successful studies on streptomycetes engineering (Fig. 2).

\section{Unique considerations for systems metabolic engineering of streptomycetes}

The established framework of systems metabolic engineering [19] can serve as a guideline for the optimal production of secondary metabolites using streptomycetes (Fig. 3). However, systems metabolic engineering of streptomycetes has additional special considerations that are not necessarily relevant to popular model organisms such as Escherichia coli and Saccharomyces cerevisiae. In this context, we first focus on these 'unique considerations' for streptomycetes, including characterization of BGCs, their encoding secondary metabolites 
and regulations involved in the secondary metabolite biosynthesis as well as molecular tools specifically adapted for the streptomycetes engineering.

\subsection{Characterization of BGCs and their secondary metabolites through computational} resources

Due to the complex nature of secondary metabolism, computational resources are needed to better understand working mechanisms of secondary metabolisms many of which are still unknown. Secondary metabolite biosynthetic pathways are also not sufficiently covered by existing general metabolic databases (e.g., KEGG and MetaCyc). Fortunately, advances in high-quality genome sequences of Streptomyces isolates (Fig. 1) and other secondary metabolite-producing organisms have sparked the development of such specialized computational resources, including databases and genome/compound mining tools, and the representative ones are presented herein. First, BGC databases allow for easy access and evaluation of curated and predicted BGCs, such as the Minimum Information on Biosynthetic Gene Clusters (MIBiG) [20], the Integrated Microbial Genomes Atlas of Biosynthetic gene Clusters (IMG-ABC) [21], and the antiSMASH database [22]. For the secondary metabolite compounds, several comprehensive databases exist as well, including NORINE [23] specifically for non-ribosomal peptides (NRPs), Antibiotic'ome [24] for the predicted molecular targets of antibiotics, and StreptomeDB [25], which presents information on compounds produced by streptomycetes.

Second, genome mining tools continue to develop with more features, which allow for predicting and assigning functions to enzymes involved in the biosynthesis of secondary metabolites. antiSMASH [12] and PRISM [14] are two of the most well-established tools, which both base their predictions on identification of signature genes or domains known to be specific for secondary metabolite BGCs [20]. Another recently released approach called EvoMining employs phylogenomic analysis to identify repurposed enzymes, originating from primary metabolism, which has been recruited to secondary metabolism for the secondary 
metabolite biosynthesis [26]. Using this EvoMining approach, Cruz-Morales et al. identified a new clade of biosynthetic enzymes involved in the biosynthesis of arseno-organic metabolites [26]. In addition, an alternative way of mining BGCs is found in the Antibiotics Resistant Target Seeker (ARTS) (https://arts2.ziemertlab.com), which evaluates BGCs based on selfresistance mechanisms of antibiotic producers [27]. ARTS scans genomes for promising BGCs based on known resistances and the presence of duplicated, co-localized housekeeping genes that display an evidence of horizontal gene transfer. In the context of systems metabolic engineering, the identified resistance genes can be used as probes to find novel BGCs without requiring detailed information on the BGC. The Gene Cluster Family (GCF) network (http://www.igb.illinois.edu/labs/metcalf/gcf/index.html) [28] is another useful approach for BGC identification, which groups gene clusters and assigns functions of previously uncharacterized BGCs.

For the compound mining, further prioritization of BGCs in promising strains can be achieved through cheminformatic analyses of the metabolome data generated from liquid chromatography (LC) tandem mass spectrometry (MS/MS) or nuclear magnetic resonance (NMR) [13,29]. Upon LC-MS/MS-guided identification of new compounds, following compound mining (or cheminformatic) tools enable automated compound characterization and dereplication: Global Alignment for natuRaL-products chemInformatiCs (GARLIC) in combination with Generalized Retrobiosynthetic Assembly Prediction Engine (GRAPE) platform [30]; and the open-access MS/MS database Global Natural Products Social Molecular Networking (GNPS) [31]. The GARLIC pipeline allows for both linking orphaned products to their corresponding BGCs and for identification of novel compounds by aligning bacterial PK and NRP BGCs predicted by PRISM to a comprehensive library of natural products from GRAPE [30]. The applicability of this pipeline consisting of PRISM, GRAPE and GARLIC was demonstrated by identifying a new compound, potensibactin, isolated from Nocardiopsis potens DSM 45234, and also by linking three previously orphaned natural products, lucensomycin, octacosaminin and bogorol, to their respective BGCs in Streptomyces achromogenes NRRL 3125, Amycolatopsis sp. NAM 50, and Brevibacillus 
laterosporus DSM 25, respectively. Meanwhile, the GNPS platform contains a comprehensive compound data collection curated by the natural product community [31]. As this community-driven knowledge-sharing database constantly expands, it allows for continuous screening of newly uploaded data sets against the established database. Besides its applicability in the discovery and characterization of natural products and their BGCs, the network can be used to identify structural analogs of a known molecule as exemplified from the analysis of a broad-spectrum antibiotic stenothricin from Streptomyces roseosporus. Based on the molecular networking and dereplication features of the platform, GNPS identified a subnetwork of five stenothricin analogs produced by Streptomyces sp. DSM5940, all with different structural properties compared to the original molecule stenothricin.

More detailed descriptions beyond these computational resources discussed herein can be found from several review papers [32-34]. In addition, to aid scientists within the field in prioritizing the individual tools, The Secondary Metabolite Bioinformatics Portal (SMBP) at http://www.secondarymetabolites.org was recently launched [35]. For each tool or database, SMBP provides the users with links and short descriptions, allowing for quick and easy browsing. SMBP can especially be useful for metabolic engineers who plan to produce secondary metabolites, but are not familiar with secondary metabolism biochemistry.

\subsection{Characterization of the regulation of secondary metabolites biosynthesis in} streptomycetes - use of omics techniques as an example

The data obtained from genome mining often provides little information on the regulation of secondary metabolites biosynthesis in streptomycetes. An additional level of information from omics techniques can be used to clarify the complex regulations involved [36]. Transcriptomics is the most accessible and most frequently adopted omics approach which monitors changes in the gene expression levels over a course of time, or as a result of external stimuli or genetic manipulation [16]. In this context, Nieselt et al. [37] conducted microarray analyses of time-point samples of S. coelicolor M145 taken during a $60 \mathrm{~h}$ fermentation, and established changes in the expression profiles of clustered genes during the metabolic switch 
from primary to secondary metabolism. Here, the general change in expression profiles observed between the $35 \mathrm{~h}$ and $36 \mathrm{~h}$ especially marked the metabolic switch, which was an interval for the depletion of phosphate from the medium. More recently, multi-omics techniques were deployed to determine the transcriptional and translational landscape of $S$. coelicolor M145 using differential RNA-sequencing (dRNA-seq) and strand-specific RNAseq (ssRNA-seq) first, followed by ribosome profiling (Ribo-seq) [38]. In particular, the use of Ribo-seq shed light on the level of translational control on genes involved in both primary and secondary metabolism, and revealed a general decline in the translation efficiency of BGCs in S. coelicolor after the cell's transition to growth phase. Furthermore, translation efficiency was negatively correlated with transcription, hence revealing the extra level of regulation to account for when embarking on the quest of strain optimization.

In addition to transcriptomics, proteomics can provide additional and complementary information on regulations associated with the transition to antibiotic production. Proteome data of S. coelicolor M145 were collected at three developmental stages ranging from compartmentalized mycelium (12 h) to the multinucleated mycelium (24 h and $72 \mathrm{~h}$ ), which revealed that hypha differentiation from substrate to aerial hypha was found to be correlated with a change in proteome composition [39]. Based on LC-MS/MS, 626 proteins were identified, of which 345 proteins were further quantified to reveal that proteins involved in primary metabolism were the most abundant in the cells in the compartmentalized mycelium, whereas proteins involved in secondary metabolism were found to predominate the cells in the multinucleated mycelium. Such omics studies will allow us better understand metabolic characteristics and complex regulations involved in biosynthesis of desired secondary metabolites.

\subsection{Molecular tools for streptomycetes engineering}

A remarkable progress recently made in the computational field has been accompanied with concurrent advances in molecular tools in order to overcome callenges associated with streptomycetes engineering: high GC contents, relatively long cultivation times and 
occasional (or frequent in some strains) reluctance towards introduction of recombinant DNA [40]. To circumvent the limitations encountered when using the standard restriction- and ligation-based cloning for genetic manipulation, the $E$. coli-derived $\lambda$ Red system was applied for faster and more efficient gene disruption, in-frame deletion, gene replacement, and refactoring of entire gene clusters in Streptomyces strains [41]. To aid genetic engineering effort even further in streptomycetes, an alternative to this classical method was reported using the S. cerevisiae I-SceI homing endonuclease for DNA double strand break (DSB)based genome editing [42,43]. This meganuclease differs from conventional restriction enzymes by its longer recognition site of 18 bp and with a lower risk of off-targets for gene editing purposes. Using a two-plasmid system, the I-SceI recognition sequence and a suitable resistance marker are introduced into a defined site on the genome of the recipient streptomycete via a single crossover event using the first plasmid. Double crossover is induced upon introduction of the second plasmid, which harbors the synthetic codonoptimized I-SceI under the control of either the constitutive ermE* or thiostrepton-inducible tipA promoter. To facilitate marker-free gene deletions, plasmids derived from pSG5 [44] with temperature sensitive replicons can be used. When using the I-SceI system for gene replacement in Streptomyces sp. Tü 6071, I-SceI-mediated homologous recombination (HR) was found to be 25 times more efficient than spontaneously occurring HR, hence, establishing its use for more systematic gene editing in streptomycetes [42].

More recently, an even more efficient and flexible system for genome engineering in streptomycetes has made its way to the field, namely the type II Clustered Regularly Interspaced Short Palindromic Repeats (CRISPR)-CRISPR associated protein (Cas9) system from Streptococcus pyogenes. The CRISPR-Cas9 technique can be applied for deletion of genes and gene clusters [45-47] in a multiplex manner [45], reversible gene expression control [47], and induction [48]. The optimized system relies on two components: the single guide RNA (sgRNA), which is a synthetic RNA consisting of a CRISPR RNA (crRNA) and trans-activating crRNA (tracrRNA) complex, and is required for guiding Cas9 to modify the targeted genome sequence; and the endonuclease Cas9 or catalytically inactive dCas9, which 
upon interaction with the sgRNA, scans the genome for protospacer adjacent motif (PAM) sequences and the corresponding 20 bp recognition site. Upon recognition of the target DNA sequence, Cas9 binds to and cleaves the specific site in the genome whereas the inactive dCas9 will bind to the target DNA without sequence modifications. Here, the dCas9 presents an easy-to-clone tool for reversible control of gene expression, and thus is useful for investigating regulation without the risk of off-target effects or subjecting the cells to the stress associated with gene replacements or mutagenesis procedures [47]. CRISPR-Cas9mediated mutagenesis can be used in the absence of templates for HR. In this case, the repair mechanism is dependent on the non-homologous end joining (NHEJ) of the DNA in Streptomyces species. However, efficiency and specificity are greatly improved if a homology template for HR is provided [45-47]. This has been demonstrated in independent studies, in which Cobb et al. [45] and Huang et al. [46] reported 60\% 100\% and 70\% 100\% efficiencies, respectively, whereas Tong et al. [47] reported near $100 \%$ efficiency for their studies on the actinorhodin gene cluster in S. coelicolor.

For specific applications, the CRISPR-Cas9 system has proven suitable for identification and examination of putative genes and BGCs for secondary metabolism in several Streptomyces species. One example is the use of the system for discovery and examination of the BGC encoding the antibiotics formicamycins in a new isolate Streptomyces formicae [49]. Using the pCRISPomyces-2 vector, which harbors both the codon-optimized Cas9 from S. pyogenes and the sgRNA cassette, the only type II polyketide synthase (PKS) BGC in S. formicae was deleted, resulting in a mutant incapable of producing any formicamycins. Using the same vector, the authors proceeded to elucidate the biosynthetic pathway by knocking out single genes in the putative BGC, and ultimately proposed a preliminary biosynthetic route for the production of this group of antibiotics [49]. Similarly, CRISPR-Cas9 was used to determine that the LuxR family cluster-situated regulator FscRI was necessary for the activation of antimycins biosynthesis in Streptomyces albus S4 [50]. Using this information, heterologous production of antimycins in S. coelicolor M1146 was achieved when the BGC was co-expressed with its activator FscRI. The general 
versatility of the CRISPR-Cas9 system has been further exemplified by its application for genome editing of rare actinomycetes, one of which is Actinoplanes sp. SE50/110 [51].

The CRISPR-Cas9 was applied in an alternative fashion for the knock-in strategy in order to activate silent BGCs in multiple Streptomyces strains [48]. Replacing native promoters with the strong, constitutive promoter kasOp* using the CRISPR-Cas9 system led to the production of new compounds in S. roseosporus, Streptomyces venezuelae, and Streptomyces viridochromogenes. Furthermore, the compound isolated from $S$. viridochromogenes was found to be a novel pigmented PK produced from an otherwise silent BGC of the type II PKS. Although this activation strategy bears striking similarity to a technique previously reported for the activation of five gene clusters in S. albus J1074 [52], the incentive to use the CRISPR-Cas9 system is the increased efficiency of HRs, presenting an improved method for genetic manipulation of strains suffering from the low efficiency of natural recombination.

The use of synthetic or natural strong promoters has also been proven useful in activating BGCs with low or no expression levels [53-55]. Based on the xylE reporter gene and RNA-seq, 32 promoters from S. albus J1034 were identified and characterized, revealing ten promoters with strengths $200 \% \sim 1,300 \%$ higher than that reported for the standard, constitutive promoter ermE*p [55]. Using a similar screening approach, Siegl et al. [54] evaluated their collection of synthetic promoters, and reported strengths of $2 \% \sim 319 \%$ in comparison with that of $e r m E^{*}$ p.

In addition to the newly adapted molecular tools discussed above, various systems metabolic engineering strategies for the model organisms can also be deployed to refactor secondary metabolites biosynthesis in native hosts, including removal of competing pathways to change the flux distribution or increase the pool of precursors, deletion of repressors, and overexpression of activators [56]. With further advances in the field, systems metabolic engineering strategies will also be employed for establishment and optimization of secondary metabolites production in heterologous hosts in the future (see below). 


\subsection{Additional approaches that facilitate streptomycetes engineering}

Besides the molecular tools discussed above, several additional approaches exist, which may further facilitate streptomycetes enginering and the secondary metabolite production. First, if the native host is reluctant towards any genome engineering efforts, heterologous expression might prove a helpful alternative [40,57]. Strains such as S. albus, S. avermitilis, S. coelicolor and Streptomyces lividans are readily amenable to cloning and expression, and therefore, their use as heterologous hosts circumvents native host limitations [58]. Also, the construction of genome-minimized hosts, which have had one or more BGCs removed from their genomes, might improve titers of a given product by avoiding the use of common precursors for other secondary metabolites [56,58]. One such example is the minimized host S. coelicolor M1154 which had four of its BGCs deleted in addition to the introduction of point mutations in $r p o B$ and $r p s L$, and is known to have an improved antibiotic production performance. Using this host, Gomez-Escribano and Bibb reported 30- and 40-fold increases in the production titers of congocidine and chloramphenicol, respectively, compared to the control strain S. coelicolor M145 as the production host [59]. An even further reduction of the S. coelicolor genome was obtained by Zhou et al. [60] who, using PCR-targeting of cosmids for the gene disruption, sequentially deleted all the ten BGCs encoding PKS and non-ribosomal peptide synthetases (NRPS) in addition to a 900-kb subtelomeric region. A series of mutants were generated as a result, one of which had $14 \%$ of its genome deleted. Furthermore, a physiological comparison of the constructed genome-minimized mutants to the wild-type showed no differences in growth rates. Hence, this strategy can provide new hosts suitable for heterologous expression of a wide variety of BGCs.

The development of a small molecule activation approach could also prove helpful when working with the troublesome isolates. In this regard, chemical elicitation which uses small molecules to induce the metabolites production in a given strain has been used with positive outcomes [61,62]. Using the GFP- and LacZ-based reporter systems to monitor activation of gene expression, a library of 640 elicitors was screened to find nine of them 
eliciting production of two otherwise silent BGCs in Burkholderia thailandensis E264 [61]. Similarly, in a study on the effects of rare earth elements on secondary metabolite production in S. coelicolor, scandium and lanthanum were found to induce gene expression for the actinorhodin BGC in addition to four otherwise silent BGCs [63]. More recently, a library of 30,569 small molecules was screened for their effects on actinorhodin production in $S$. coelicolor M145 [64]. This led to the isolation of a group of four antibiotic-remodeling compounds (ARCs), of which ARC2 was found capable of eliciting secondary metabolism in related Streptomyces species as well. Following these findings, the synthetic derivative $\mathrm{Cl}$ ARC was used for the stronger elicitation of BGCs encoding molecules at a low abundance in a panel of fifty Actinomycete strains [65]. This resulted in the identification of several CIARC-induced compounds including oxohygrolidin from Streptomyces ghanaensis, not previously known to produce this molecule [66]. Furthermore, based on bioactivity-guided assays, it was shown that while the extracts of the elicitor-treated S. ghanaensis failed to provide detectable inhibition, purified oxohydrolidin displayed activity against $S$. cerevisiae Y7092. The latter finding provides strong incentives to focus future work on purified compounds in order to avoid masking of low abundance molecules in complex sample mixtures. Furthermore, such systematic analyses show a great promise in high-throughput screening approaches in that they allow for both screening multiple elicitors on one strain, or, as reported by Craney et al. [64], screening one elicitor molecule against a collection of strains. The major limitation to such an approach, however, remains in the massive amount of samples generated, which all require time-consuming subsequent analytics studies.

\section{Recent examples of systems metabolic engineering of streptomycetes for the optimized production of secondary metabolites}

The framework of systems metabolic engineering continues to be adopted to improve the production of secondary metabolites in streptomycetes, as seen from the many recent successful examples reported (Table 1). Some noteworthy examples include the production of 
tacrolimus (FK506) using Streptomyces tsukubaensis [67-69], ascomycin (FK520) using Streptomyces hygroscopicus var. ascomyceticus [70-72], avermectin using S. avermitilis [73], and pristinamycin I (PI) and II (PII) using Streptomyces pristinaespiralis [74,75].

To solve the issue of low production yield of tacrolimus in the natural producer, Wang et al. [67] first determined the intracellular response of S. tsukubaensis to exogenous feeding of four precursors known to promote the tacrolimus biosynthesis. Using a weighted correlation network analysis (WGCNA) on a dataset containing 93 different intracellular metabolites measured with gas chromatography (GC)-MS and LC-MS/MS, metabolites highly associated with the tacrolimus biosynthesis were identified. Furthermore, time-point sampling led to the identification of three pronounced pathways involved in the tacrolimus biosynthesis, namely pentose phosphate, shikimate, and aspartate pathways. Using this information and the GSMM of S. tsukubaensis, the genes aroC and dapA, involved in the biosynthesis of chorismate and lysine, respectively, were identified for overexpression, and the effects of overexpression of these genes were experimentally validated. As a result, a mutant overexpressing the $\operatorname{aroC}$ and $\operatorname{dapA}$ genes produced 1.64-fold higher yields of tacrolimus, compared to the wild-type strain.

Ascomycin, an ethyl analog of tacrolimus, is another important immunosuppressant. To optimize the production of this compound, the natural producer S. hygroscopicus var. ascomyceticus has undergone several rounds of metabolic engineering. Early strain development efforts have so far included femtosecond laser irradiation mutagenesis combined with a shikimic acid enduring screening. Although shikimic acid was found to have a positive effect on the ascomycin production, the mutant strain FS35, from the mutagenesis screen, also displayed high sensitivy towards this substrate. To overcome this negative effect, an endurance screening with shikimic acid was carried out, resulting in the isolation of a shikimic acid-resistant mutant strain SA68 with ascomycin yield of $330 \mathrm{mg} / \mathrm{L}$ (270 mg/L for the parent strain) [70,71]. More recently, systems metabolic engineering [72] was employed to obtain a high-yield ascomycin producing strain, which showed $84.8 \%$ titer improvement, compared to the parent strain. Prior to the strain engineering, ${ }^{13} \mathrm{C}$-labelling experiments and 
metabolic flux analysis (MFA) were employed in parallel to construct and validate an ascomycin metabolic network. Elementary flux mode analysis (EFMA) on the model allowed the identification of chorismatase FkbO and pyruvate carboxylase Pyc as overexpression and inactivation targets, respectively. Furthermore, from the fed-batch fermentation of the highproducer strain TD- $\Delta$ Pyc-FkbO, ethylmalonyl-CoA was found to be limited towards the end of fermentation, which opens up for future engineering efforts.

Similarly, industrial producer strains have undergone extensive systems metabolic engineering to improve their production titers of a group of avermectins [73]. As avermectin biosynthesis is under the tight control of several regulators, much focus has been put on relieving such regulators. The pathway-specific regulator AveR, which is involved in the activation of avermectin biosynthesis, is itself subject to both negative and positive regulation [73]. Here, the two TetR family transcriptional regulators (TFRs) encoded by the genes SAV576 and SAV577 were found to indirectly downregulate the avermectin biosynthesis through binding to the promoter region of SAV575; SAV575 is known to be involved in providing acetate and propionate extender units required for the avermectin production [76,77]. Using a combination of microarray analysis, genetic studies, and chromatin immunoprecipitation (ChIP) assays, the expression levels of the TFRs and SAV575 were determined for both mutant and wild-type strains as well as the binding sites of SAV575 for the TFRs. This observation allowed for proposing the regulatory role of the TFRs in the avermectin production. Based on this information, a double deletion mutant ( $\triangle$ SAV576 and $\Delta$ SAV577) was constructed, which showed $\sim 3$ fold higher production of avermectin, compared to the wild-type [77].

A more recent example of using a combinatorial metabolic engineering approach has been presented for the production of streptogramin-like antibiotic pristinamycin, which consists of two chemically unrelated compounds PI and PII [78]. As the native host $S$. pristinaespiralis suffers from low yields, additional copies of the two BGCs responsible for the production of PI and PII were introduced in addition to the deletion of repressors [74,75]. The metabolic engineering efforts were strongly aided from previous findings of the 
regulatory cascade governing the biosynthesis of both PI and PII in S. pristinaespiralis [79]. For PII, the deletion of the two cluster-specific repressors PapR3 and PapR5 along with overexpression of the activators PapR4 and PapR6 resulted in a 1.5-fold higher production of PII in a mutant harboring an additional copy of the PII BGC, compared to the wild-type. Furthermore, the addition of resins to relieve both feedback inhibition and toxicity of PII resulted in the 5.26-fold higher production, compared to wild-type, when grown in $5 \mathrm{~L}$ bioreactors [74]. For PI, the highest production was observed in a mutant in which the BGC of PII was removed, an additional copy of the PI BGC introduced, and the repressor PapR3 deleted. Besides the 2.4-fold increased production of PI compared to the wild-type, interestingly, the mutant with only the PII BGC removed showed 20-40\% lowered PI production, compared to the wild-type, revealing a possible role of PII as a coactivator or inducer of PI production [75]. Further characterization efforts are needed to unravel the regulatory mechanism behind this observation and to additionally engineer the strain accordingly.

Additional examples on the use of both metabolic engineering and more general engineering strategies for the optimization of secondary metabolites production in actinobacteria have recently been reviewed elsewhere [56].

\section{Conclusions}

The expansions and improvements witnessed within the fields of genome and compound mining, omics, and molecular cloning techniques are paving the way for systems metabolic engineering to harness the production potential of streptomycetes. Systematic and global analyses that have been undertaken will continue to improve, and, as a result, will foster faster and better decision making when re-designing a given strain for the optimal production of a target secondary metabolite and discovery of new secondary metabolites. However, it should be noted that, despite all these resources available, a certain amount of iterations of the design-build-test-learn cycle are still necessary as demonstrated for other microbial metabolic 
engineering cases, especially taking into account the complex regulation between primary and secondary metabolism in streptomycetes. Implementation of molecular cloning methods in an automated, high-throughput setting, such as iBioFab [80], biosensors for detecting expression of secondary metabolite biosynthesis genes [81], or biosensors for the production of a desired secondary metabolite [82] might further reduce time and efforts needed to optimize the production of secondary metabolites using streptomycetes. It is expected that the tools and strategies of systems metabolic engineering of streptomycetes will advance rapidly to harness full biotechnological potentials of this important class of bacteria.

\section{Acknowledgement}

This work was funded by a grant from the Novo Nordisk Foundation. H.U.K and S.Y.L. are also supported by the Technology Development Program to Solve Climate Changes on Systems Metabolic Engineering for Biorefineries (NRF-2012M1A2A2026556 and NRF2012M1A2A2026557) from the Ministry of Science and ICT through the National Research Foundation (NRF) of Korea.

\section{Conflict of interest}

The authors declare no financial or commercial conflict of interest.

\section{References}

1. A. L. Demain and S. Sanchez, J. Antibiot. (Tokyo)., 2009, 62, 1.

2. J. Bérdy, J. Antibiot. , 2005, 58, 1.

3. S. D. Bentley, K. F. Chater, A.-M. Cerdeño-Tárraga, G. L. Challis, N. R. Thomson, K. D. James, D. E. Harris, M. A. Quail, H. Kieser, D. Harper, A. Bateman, S. Brown, G. Chandra, C. W. Chen, M. Collins, A. Cronin, A. Fraser, A. Goble, J. Hidalgo, T. Hornsby, S. Howarth, C.-H. Huang, T. Kieser, L. Larke, L. Murphy, K. Oliver, S. O’Neil, E. Rabbinowitsch, M.-A. Rajandream, K. Rutherford, S. Rutter, K. Seeger, D. Saunders, S. Sharp, R. Squares, S. Squares, K. Taylor, T. Warren, A. Wietzorrek, J. Woodward, B. G. Barrell, J. Parkhill, and D. A. Hopwood, Nature, 2002, 417, 6885. 
4. H. Ikeda, J. Ishikawa, A. Hanamoto, M. Shinose, H. Kikuchi, T. Shiba, Y. Sakaki, M. Hattori, and S. Ōmura, Nat. Biotechnol., 2003, 21, 5.

5. F. Barona-Gómez, U. Wong, A. E. Giannakopulos, P. J. Derrick, and G. L. Challis, J. Am. Chem. Soc., 2004, 126, 50.

6. G. L. Challis and J. Ravel, FEMS Microbiol. Lett., 2000, 187, 2.

7. S. Lautru, R. J. Deeth, L. M. Bailey, and G. L. Challis, Nat. Chem. Biol., 2005, 1, 5.

8. B. Zhao, X. Lin, L. Lei, D. C. Lamb, S. L. Kelly, M. R. Waterman, and D. E. Cane, J. Biol. Chem., 2008, 283, 13.

9. J. P. Gomez-Escribano, L. Song, D. J. Fox, V. Yeo, M. J. Bibb, and G. L. Challis, Chem. Sci., 2012, 3, 9.

10. M. H. Medema, K. Blin, P. Cimermancic, V. de Jager, P. Zakrzewski, M. A.

Fischbach, T. Weber, E. Takano, and R. Breitling, Nucleic Acids Res., 2011, 39, Web Server issue.

11. T. Weber, K. Blin, S. Duddela, D. Krug, H. U. Kim, R. Bruccoleri, S. Y. Lee, M. A. Fischbach, R. Müller, W. Wohlleben, R. Breitling, E. Takano, and M. H. Medema, Nucleic Acids Res., 2015, 43, W1.

12. K. Blin, T. Wolf, M. G. Chevrette, X. Lu, C. J. Schwalen, S. A. Kautsar, H. G. Suarez Duran, E. L. C. de los Santos, H. U. Kim, M. Nave, J. S. Dickschat, D. A. Mitchell, E. Shelest, R. Breitling, E. Takano, S. Y. Lee, T. Weber, and M. H. Medema, Nucleic Acids Res., 2017, 45, W1.

13. M. A. Skinnider, C. A. Dejong, P. N. Rees, C. W. Johnston, H. Li, A. L. H. Webster, M. A. Wyatt, and N. A. Magarvey, Nucleic Acids Res., 2015, 43, 20.

14. M. A. Skinnider, N. J. Merwin, C. W. Johnston, and N. A. Magarvey, Nucleic Acids Res., 2017, 45, W1.

15. K.-S. Hwang, H. U. Kim, P. Charusanti, B. Ø. Palsson, and S. Y. Lee, Biotechnol. Adv., 2014, 32, 2.

16. G. P. van Wezel and K. J. McDowall, Nat. Prod. Rep., 2011, 28, 7.

17. H. U. Kim, P. Charusanti, S. Y. Lee, and T. Weber, Nat. Prod. Rep., 2016, 33, 8.

18. L. B. Pickens, Y. Tang, and Y.-H. Chooi, Annu. Rev. Chem. Biomol. Eng., 2011, 2.

19. S. Y. Lee and H. U. Kim, Nat. Biotechnol., 2015, 33, 10.

20. M. H. Medema, R. Kottmann, P. Yilmaz, M. Cummings, J. B. Biggins, K. Blin, I. de Bruijn, Y. H. Chooi, J. Claesen, R. C. Coates, P. Cruz-Morales, S. Duddela, S. Düsterhus, D. J. Edwards, D. P. Fewer, N. Garg, C. Geiger, J. P. Gomez-Escribano, A. Greule, M. Hadjithomas, A. S. Haines, E. J. N. Helfrich, M. L. Hillwig, K. Ishida, A. C. Jones, C. S. Jones, K. Jungmann, C. Kegler, H. U. Kim, P. Kötter, D. Krug, J. Masschelein, A. V Melnik, S. M. Mantovani, E. A. Monroe, M. Moore, N. Moss, H.W. Nützmann, G. Pan, A. Pati, D. Petras, F. J. Reen, F. Rosconi, Z. Rui, Z. Tian, N. J. Tobias, Y. Tsunematsu, P. Wiemann, E. Wyckoff, X. Yan, G. Yim, F. Yu, Y. Xie, B. Aigle, A. K. Apel, C. J. Balibar, E. P. Balskus, F. Barona-Gómez, A. Bechthold, H. B. Bode, R. Borriss, S. F. Brady, A. A. Brakhage, P. Caffrey, Y.-Q. Cheng, J. Clardy, R. J. Cox, R. De Mot, S. Donadio, M. S. Donia, W. A. van der Donk, P. C. Dorrestein, S. Doyle, A. J. M. Driessen, M. Ehling-Schulz, K.-D. Entian, M. A. Fischbach, L. Gerwick, W. H. Gerwick, H. Gross, B. Gust, C. Hertweck, M. Höfte, S. E. Jensen, J. Ju, L. Katz, L. Kaysser, J. L. Klassen, N. P. Keller, J. Kormanec, O. P. Kuipers, T. Kuzuyama, N. C. Kyrpides, H.-J. Kwon, S. Lautru, R. Lavigne, C. Y. Lee, B. Linquan, et al., Nat. Chem. Biol., 2015, 11, 9.

21. M. Hadjithomas, I.-M. A. Chen, K. Chu, A. Ratner, K. Palaniappan, E. Szeto, J. Huang, T. B. K. Reddy, P. Cimermančič, M. A. Fischbach, N. N. Ivanova, V. M. 
Markowitz, N. C. Kyrpides, and A. Pati, MBio, 2015, 6, 4.

22. K. Blin, M. H. Medema, R. Kottmann, S. Y. Lee, and T. Weber, Nucleic Acids Res., 2017, 45, D1.

23. A. Flissi, Y. Dufresne, J. Michalik, L. Tonon, S. Janot, L. Noé, P. Jacques, V. Leclère, and M. Pupin, Nucleic Acids Res., 2016, 44, D1.

24. C. W. Johnston and N. A. Magarvey, Nat. Chem. Biol., 2015, 11, 3.

25. D. Klementz, K. Döring, X. Lucas, K. K. Telukunta, A. Erxleben, D. Deubel, A.

Erber, I. Santillana, O. S. Thomas, A. Bechthold, and S. Günther, Nucleic Acids Res., 2015, 44, D1.

26. P. Cruz-Morales, J. F. Kopp, C. Martínez-Guerrero, L. A. Yáñez-Guerra, N. SelemMojica, H. Ramos-Aboites, J. Feldmann, and F. Barona-Gómez, Genome Biol. Evol., 2016, 8, 6 .

27. M. Alanjary, B. Kronmiller, M. Adamek, K. Blin, T. Weber, D. Huson, B. Philmus, and N. Ziemert, Nucleic Acids Res., 2017, 45, W1.

28. J. R. Doroghazi, J. C. Albright, A. W. Goering, K.-S. Ju, R. R. Haines, K. A. Tchalukov, D. P. Labeda, N. L. Kelleher, and W. W. Metcalf, Nat. Chem. Biol., 2014, $10,11$.

29. C. Wu, H. K. Kim, G. P. van Wezel, and Y. H. Choi, Drug Discov. Today Technol., 2015, 13.

30. C. A. Dejong, G. M. Chen, H. Li, C. W. Johnston, M. R. Edwards, P. N. Rees, M. A. Skinnider, A. L. H. Webster, and N. A. Magarvey, Nat. Chem. Biol., 2016, 12, 12.

31. M. Wang, J. J. Carver, V. V Phelan, L. M. Sanchez, N. Garg, Y. Peng, D. D. Nguyen, J. Watrous, C. A. Kapono, T. Luzzatto-Knaan, C. Porto, A. Bouslimani, A. V Melnik, M. J. Meehan, W.-T. Liu, M. Crüsemann, P. D. Boudreau, E. Esquenazi, M. SandovalCalderón, R. D. Kersten, L. A. Pace, R. A. Quinn, K. R. Duncan, C.-C. Hsu, D. J. Floros, R. G. Gavilan, K. Kleigrewe, T. Northen, R. J. Dutton, D. Parrot, E. E. Carlson, B. Aigle, C. F. Michelsen, L. Jelsbak, C. Sohlenkamp, P. Pevzner, A. Edlund, J. McLean, J. Piel, B. T. Murphy, L. Gerwick, C.-C. Liaw, Y.-L. Yang, H.-U. Humpf, M. Maansson, R. A. Keyzers, A. C. Sims, A. R. Johnson, A. M. Sidebottom, B. E. Sedio, A. Klitgaard, C. B. Larson, C. A. Boya P, D. Torres-Mendoza, D. J. Gonzalez, D. B. Silva, L. M. Marques, D. P. Demarque, E. Pociute, E. C. O’Neill, E. Briand, E. J. N. Helfrich, E. A. Granatosky, E. Glukhov, F. Ryffel, H. Houson, H. Mohimani, J. J. Kharbush, Y. Zeng, J. A. Vorholt, K. L. Kurita, P. Charusanti, K. L. McPhail, K. F. Nielsen, L. Vuong, M. Elfeki, M. F. Traxler, N. Engene, N. Koyama, O. B. Vining, R. Baric, R. R. Silva, S. J. Mascuch, S. Tomasi, S. Jenkins, V. Macherla, T. Hoffman, V. Agarwal, P. G. Williams, J. Dai, R. Neupane, J. Gurr, A. M. C. Rodríguez, A. Lamsa, C. Zhang, K. Dorrestein, B. M. Duggan, J. Almaliti, et al., Nat. Biotechnol., 2016, 34, 8.

32. T. Weber, Int. J. Med. Microbiol., 2014, 304, 3-4.

33. M. H. Medema and M. A. Fischbach, Nat. Chem. Biol., 2015, 11, 9.

34. N. Ziemert, M. Alanjary, and T. Weber, Nat. Prod. Rep., 2016, 33, 8.

35. T. Weber, K. Blin, and H. U. Kim, Synth. Syst. Biotechnol., 2016, 1, 2.

36. A. K. Chaudhary, D. Dhakal, and J. K. Sohng, Biomed Res. Int., 2013, 3, 8.

37. K. Nieselt, F. Battke, A. Herbig, P. Bruheim, A. Wentzel, Ø. M. Jakobsen, H. Sletta, M. T. Alam, M. E. Merlo, J. Moore, W. A. M. Omara, E. R. Morrissey, M. A. JuarezHermosillo, A. Rodríguez-García, M. Nentwich, L. Thomas, M. Iqbal, R. Legaie, W. H. Gaze, G. L. Challis, R. C. Jansen, L. Dijkhuizen, D. A. Rand, D. L. Wild, M. Bonin, J. Reuther, W. Wohlleben, M. C. M. Smith, N. J. Burroughs, J. F. Martín, D. 
A. Hodgson, E. Takano, R. Breitling, T. E. Ellingsen, and E. M. H. Wellington, BMC Genomics, 2010, 11.

38. Y. Jeong, J.-N. Kim, M. W. Kim, G. Bucca, S. Cho, Y. J. Yoon, B.-G. Kim, J.-H. Roe, S. C. Kim, C. P. Smith, and B.-K. Cho, Nat. Commun., 2016, 7.

39. A. Manteca, J. Sanchez, H. R. Jung, V. Schwämmle, and O. N. Jensen, Mol. Cell. Proteomics, 2010, 9, 7.

40. M. M. Zhang, Y. Wang, E. L. Ang, and H. Zhao, Nat. Prod. Rep., 2016, 33, 8.

41. B. Gust, G. Chandra, D. Jakimowicz, T. Yuqing, C. J. Bruton, and K. F. Chater, Adv. Appl. Microbiol., 2004, 54.

42. T. Siegl, L. Petzke, E. Welle, and A. Luzhetskyy, Appl. Microbiol. Biotechnol., 2010, 87, 4.

43. L. T. Fernández-Martínez and M. J. Bibb, Sci. Rep., 2014, 4.

44. G. Muth, B. Nußbaumer, W. Wohlleben, and A. Pühler, MGG Mol. Gen. Genet., 1989, 219, 3.

45. R. E. Cobb, Y. Wang, and H. Zhao, ACS Synth. Biol., 2015, 4, 6.

46. H. Huang, G. Zheng, W. Jiang, H. Hu, and Y. Lu, Acta Biochim. Biophys. Sin. (Shanghai)., 2015, 47, 4.

47. Y. Tong, P. Charusanti, L. Zhang, T. Weber, and S. Y. Lee, ACS Synth. Biol., 2015, 4, 9.

48. M. M. Zhang, F. T. Wong, Y. Wang, S. Luo, Y. H. Lim, E. Heng, W. L. Yeo, R. E. Cobb, B. Enghiad, E. L. Ang, and H. Zhao, Nat. Chem. Biol., 2017, 13, 6.

49. Z. Qin, J. T. Munnoch, R. Devine, N. A. Holmes, R. F. Seipke, K. A. Wilkinson, B. Wilkinson, and M. I. Hutchings, Chem. Sci., 2017, 8, 4.

50. T. C. McLean, P. A. Hoskisson, and R. F. Seipke, mSphere, 2016, 1, 6.

51. T. Wolf, T. Gren, E. Thieme, D. Wibberg, T. Zemke, A. Pühler, and J. Kalinowski, J. Biotechnol., 2016, 231.

52. C. Olano, I. García, A. González, M. Rodriguez, D. Rozas, J. Rubio, M. SánchezHidalgo, A. F. Braña, C. Méndez, and J. A. Salas, Microb. Biotechnol., 2014, 7, 3.

53. Y. Luo, H. Huang, J. Liang, M. Wang, L. Lu, Z. Shao, R. E. Cobb, and H. Zhao, Nat. Commun., 2013, 4.

54. T. Siegl, B. Tokovenko, M. Myronovskyi, and A. Luzhetskyy, Metab. Eng., 2013, 19.

55. Y. Luo, L. Zhang, K. W. Barton, and H. Zhao, ACS Synth. Biol., 2015, 4, 9.

56. O. Bilyk and A. Luzhetskyy, Curr. Opin. Biotechnol., 2016, 42.

57. Y. Luo, B. Enghiad, and H. Zhao, Nat. Prod. Rep., 2016, 33, 2.

58. R. H. Baltz, J. Ind. Microbiol. Biotechnol., 2016, 43, $2-3$.

59. J. P. Gomez-Escribano and M. J. Bibb, Microb. Biotechnol., 2011, 4, 2.

60. M. Zhou, X. Jing, P. Xie, W. Chen, T. Wang, H. Xia, and Z. Qin, FEMS Microbiol. Lett., 2012, 333, 2.

61. M. R. Seyedsayamdost, Proc. Natl. Acad. Sci., 2014, 111, 20.

62. U. R. Abdelmohsen, T. Grkovic, S. Balasubramanian, M. S. Kamel, R. J. Quinn, and U. Hentschel, Biotechnol. Adv., 2015, 33, 6.

63. Y. Tanaka, T. Hosaka, and K. Ochi, J. Antibiot. , 2010, 63, 8.

64. A. Craney, C. Ozimok, S. M. Pimentel-Elardo, A. Capretta, and J. R. Nodwell, Chem. Biol., 2012, 19, 8. 
65. M. N. Thaker, W. Wang, P. Spanogiannopoulos, N. Waglechner, A. M. King, R. Medina, and G. D. Wright, Nat. Biotechnol., 2013, 31, 10.

66. S. M. Pimentel-Elardo, D. Sørensen, L. Ho, M. Ziko, S. A. Bueler, S. Lu, J. Tao, A. Moser, R. Lee, D. Agard, G. Fairn, J. L. Rubinstein, B. K. Shoichet, and J. R. Nodwell, ACS Chem. Biol., 2015, 10, 11.

67. C. Wang, J. Liu, H. Liu, S. Liang, and J. Wen, J. Ind. Microbiol. Biotechnol., 2017, 44, 11.

68. C. Wang, J. Liu, H. Liu, J. Wang, and J. Wen, Biochem. Eng. J., 2017, 123.

69. J. Wang, H. Liu, D. Huang, L. Jin, C. Wang, and J. Wen, Appl. Microbiol. Biotechnol., 2017, 101, 6.

70. H. Qi, X. Xin, S. Li, J. Wen, Y. Chen, and X. Jia, Biotechnol. Bioprocess Eng., 2012, $17,4$.

71. H. Qi, S. Zhao, J. Wen, Y. Chen, and X. Jia, Biochem. Eng. J., 2014, 82.

72. H. Qi, M. Lv, K. Song, and J. Wen, Biotechnol. Bioeng., 2017, 114, 5.

73. J. Chen, M. Liu, X. Liu, J. Miao, C. Fu, H. Gao, R. Müller, Q. Zhang, and L. Zhang, Synth. Syst. Biotechnol., 2016, 1, 1.

74. L. Li, Y. Zhao, L. Ruan, S. Yang, M. Ge, W. Jiang, and Y. Lu, Metab. Eng., 2015, 29.

75. J. Meng, R. Feng, G. Zheng, M. Ge, Y. Mast, W. Wohlleben, J. Gao, W. Jiang, and Y. Lu, Synth. Syst. Biotechnol., 2017, $2,2$.

76. J. Guo, X. Zhang, S. Luo, F. He, Z. Chen, Y. Wen, and J. Li, PLoS One, 2013, 8, 8.

77. J. Guo, X. Zhang, Z. Chen, Y. Wen, and J. Li, PLoS One, 2014, 9, 6.

78. Y. Mast, T. Weber, M. Gölz, R. Ort-Winklbauer, A. Gondran, W. Wohlleben, and E. Schinko, Microb. Biotechnol., 2010, 4, 2.

79. Y. Mast, J. Guezguez, F. Handel, and E. Schinko, Appl. Environ. Microbiol., 2015, 81, 19.

80. R. Chao, J. Liang, I. Tasan, T. Si, L. Ju, and H. Zhao, ACS Synth. Biol., 2017, 6, 4.

81. F. Guo, S. Xiang, L. Li, B. Wang, J. Rajasärkkä, K. Gröndahl-Yli-Hannuksela, G. Ai, M. Metsä-Ketelä, and K. Yang, Metab. Eng., 2015, 28.

82. Y.-Q. Sun, T. Busche, C. Rückert, C. Paulus, Y. Rebets, R. Novakova, J. Kalinowski, A. Luzhetskyy, J. Kormanec, O. N. Sekurova, and S. B. Zotchev, ACS Synth. Biol., 2017, 6, 6.

83. I. Borodina, P. Krabben, and J. Nielsen, Genome Res., 2005, 15, 6.

84. I. Borodina, J. Siebring, J. Zhang, C. P. Smith, G. van Keulen, L. Dijkhuizen, and J. Nielsen, J. Biol. Chem., 2008, 283, 37.

85. M. Kim, J. Sang Yi, J. Kim, J.-N. Kim, M. W. Kim, and B.-G. Kim, Biotechnol. J., 2014, 9, 9 .

86. D. Huang, X. Jia, J. Wen, G. Wang, G. Yu, Q. Caiyin, and Y. Chen, Appl. Biochem. Biotechnol., 2011, 165, 7-8.

87. D. Huang, J. Wen, G. Wang, G. Yu, X. Jia, and Y. Chen, Appl. Microbiol. Biotechnol., 2012, 94, 3.

88. L. Dang, J. Liu, C. Wang, H. Liu, and J. Wen, J. Ind. Microbiol. Biotechnol., 2017, 44, 2.

89. G.-Y. Tan, K. Deng, X. Liu, H. Tao, Y. Chang, J. Chen, K. Chen, Z. Sheng, Z. Deng, and T. Liu, ACS Synth. Biol., 2017, 6, 6. 
Tables

Table 1 Examples of metabolic engineering strategies used for the optimization of secondary metabolite production in streptomycete hosts

\begin{tabular}{|c|c|c|c|c|}
\hline Secondary metabolite & Streptomyces host & Metabolic engineering strategies & $\begin{array}{l}\text { Highest yield } \\
\text { increase } \\
\text { (compared to } \\
\text { control strain) } \\
\end{array}$ & References \\
\hline Actinorhodin & Streptomyces coelicolor A3(2) & $\begin{array}{l}\text { - Construction of two comprehensive GSMMs: iIB711 and iMK1208. The latest } \\
\text { iMK1208 comprises } 1208 \text { genes, } 1643 \text { reactions, and } 1246 \text { metabolites } \\
\text { - Construction of a mutant strain with improved yields } \\
\text { - }{ }^{13} \text { C-MFA and transcriptional analysis of mutant and parental strains to compare } \\
\text { their fluxes and gene expression patterns, respectively } \\
\text { - FSEOF on iMK1208 to identify additional gene target for overexpression and } \\
\text { subsequent experimental verification }\end{array}$ & 52-fold & [83-85] \\
\hline Ascomycin & $\begin{array}{l}\text { Streptomyces hygroscopicus } \\
\text { var. ascomyceticus }\end{array}$ & $\begin{array}{l}\text { - Parallel }{ }^{13} \mathrm{C} \text { labeling and MFA to construct metabolic network model } \\
\text { - EFMA on the ascomycin network model for target predictions } \\
\text { - Strain engineering by overexpression and inactivation of genes } \\
\text { - Addition of resin HP20 in the growth medium }\end{array}$ & $84.8 \%$ & {$[70-72]$} \\
\hline Avermectin & Streptomyces avermitilis & $\begin{array}{l}\text { - Increasing flux through precursor pathways } \\
\text { - Microarray analysis and ChIP assays for the analysis of expression levels } \\
\text { - Identification and deletion of regulators with inhibitory effect on production }\end{array}$ & 3-fold & {$[73,76,77]$} \\
\hline Daptomycin & $\begin{array}{l}\text { Streptomyces roseosporus } \\
\text { LC-511 }\end{array}$ & $\begin{array}{l}\text { - MFA to identify potential bottlenecks in biosynthesis } \\
\text { - Identification of three genes for overexpression to increase fluxes through rate- } \\
\text { limiting pathway } \\
\text { - Construction of a triple overexpression mutant with improved titers } \\
\text { - Transcriptional analysis to evaluate gene expression patterns of parental and } \\
\text { mutant strains } \\
\text { - Addition of glucose to fed-batch fermentations of the triple mutant strain to } \\
\text { improve yields further }\end{array}$ & $43.2 \%$ & {$[86,87]$} \\
\hline Pristinamycin & Streptomyces pristinaespiralis & $\begin{array}{l}\text { - Transcriptional analysis by RT-PCR for the evaluation of regulatory network } \\
\text { involved in biosynthesis } \\
\text { - Strain engineering by introduction of extra copies of the BGC, deletion of } \\
\text { repressors, and overexpression of activators } \\
\text { - Addition of resins to bioreactors }\end{array}$ & $\begin{array}{c}\text { 2.4-fold (PI) } \\
\text { 5.26-fold (PII) }\end{array}$ & {$[74,75]$} \\
\hline
\end{tabular}




\begin{tabular}{|c|c|c|c|c|}
\hline Rapamycin & $\begin{array}{c}\text { Streptomyces hygroscopicus } \\
\text { ATCC } 29253\end{array}$ & $\begin{array}{l}\text { - Construction of a GSMM with } 1003 \text { reactions and } 711 \text { metabolites } \\
\text { - FBA and MOMA analyses to evaluate gene targets for improving production } \\
\text { - Construction of a mutant strain with one gene knock-out and overexpression of } \\
\text { two genes showing higher fluxes through key primary metabolism pathways }\end{array}$ & $142.3 \%$ & [88] \\
\hline Spinosad & Streptomyces albus J1074 & $\begin{array}{l}\text { - Construction of a Saccharopolyspora spinosa NRRL } 18395 \text { bacterial artificial } \\
\text { chromosome library } \\
\text { - Establishment of heterologous expression of spinosyn BGC in Streptomyces } \\
\text { lividans TK24 and S. albus J1074 } \\
\text { - Transcriptional and translational (proteomics) analyses to evaluate heterologous } \\
\text { and native hosts } \\
\text { - Metabolomics to identify the best suited candidate host for the expression of } \\
\text { spinosyn BGC } \\
\text { - Pathway refactoring including promoter engineering and overexpression of } \\
\text { synthetic modules in S. albus mutant }\end{array}$ & 1000-fold & [89] \\
\hline Tacrolimus & Streptomyces tsukubaensis & $\begin{array}{l}\text { - Proteomic and metabolomic analyses of an overproducer strain fed with soybean } \\
\text { oil } \\
\text { - WGCNA for the identification of pronounced precursor pathways } \\
\text { - Identification of key limiting steps in the pronounced pathways using a GSMM } \\
\text { - Gene overexpression to increase fluxes through two precursor pathways }\end{array}$ & 1.64-fold & [67-69] \\
\hline
\end{tabular}




\section{Figure legends}

Figure 1. Cumulative number of whole genome sequences of Streptomyces species published in National Center for Biotechnology Information (NCBI) from 2001 to September 2017.

Figure 2. Schematic representation of the workflow of systems metabolic engineering for streptomycetes. Characterization of BGCs, their encoding secondary metabolites and biosynthesis regulations involved is critical in engineering streptomycetes for the production of novel compounds and the higher yields of a known end product or. Genome and compound mining, omics techniques, molecular tools as well as the use of elicitors play important roles in this process.

Figure 3. Workflow of systems metabolic engineering for the optimal production of secondary metabolites. The workflow shown includes tools and methods used within the individual steps. The workflow can be applied in an iterative fashion as illustrated by the design-build-test-learn cycle. The use of the entire workflow is exemplified by the production of ascomycin using Streptomyces hygroscopicus var. ascomyceticus. Here, (1) and (2) illustrates the two separate rounds of engineering applied for the optimization of ascomycin production. 


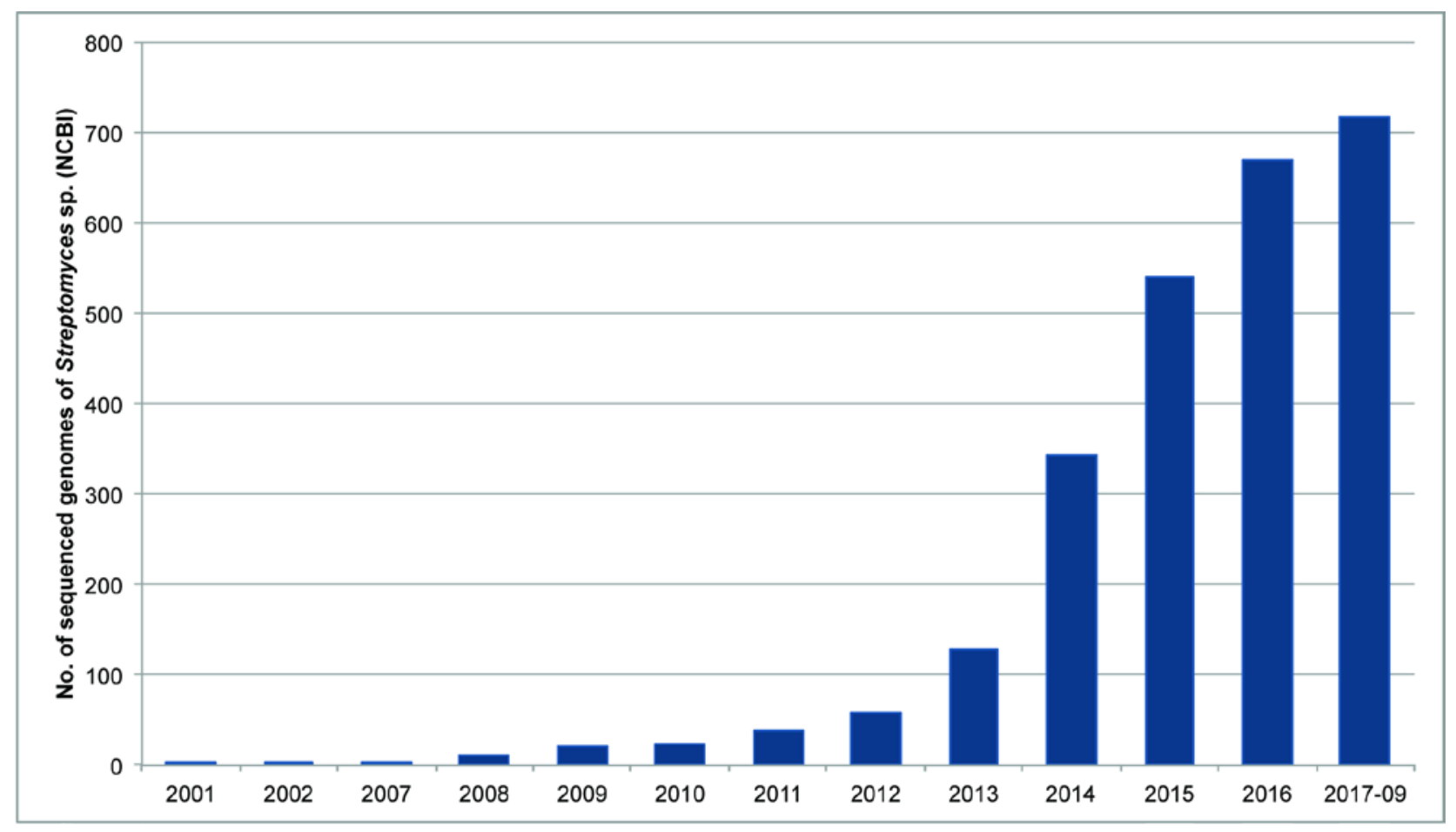

Figure. 1

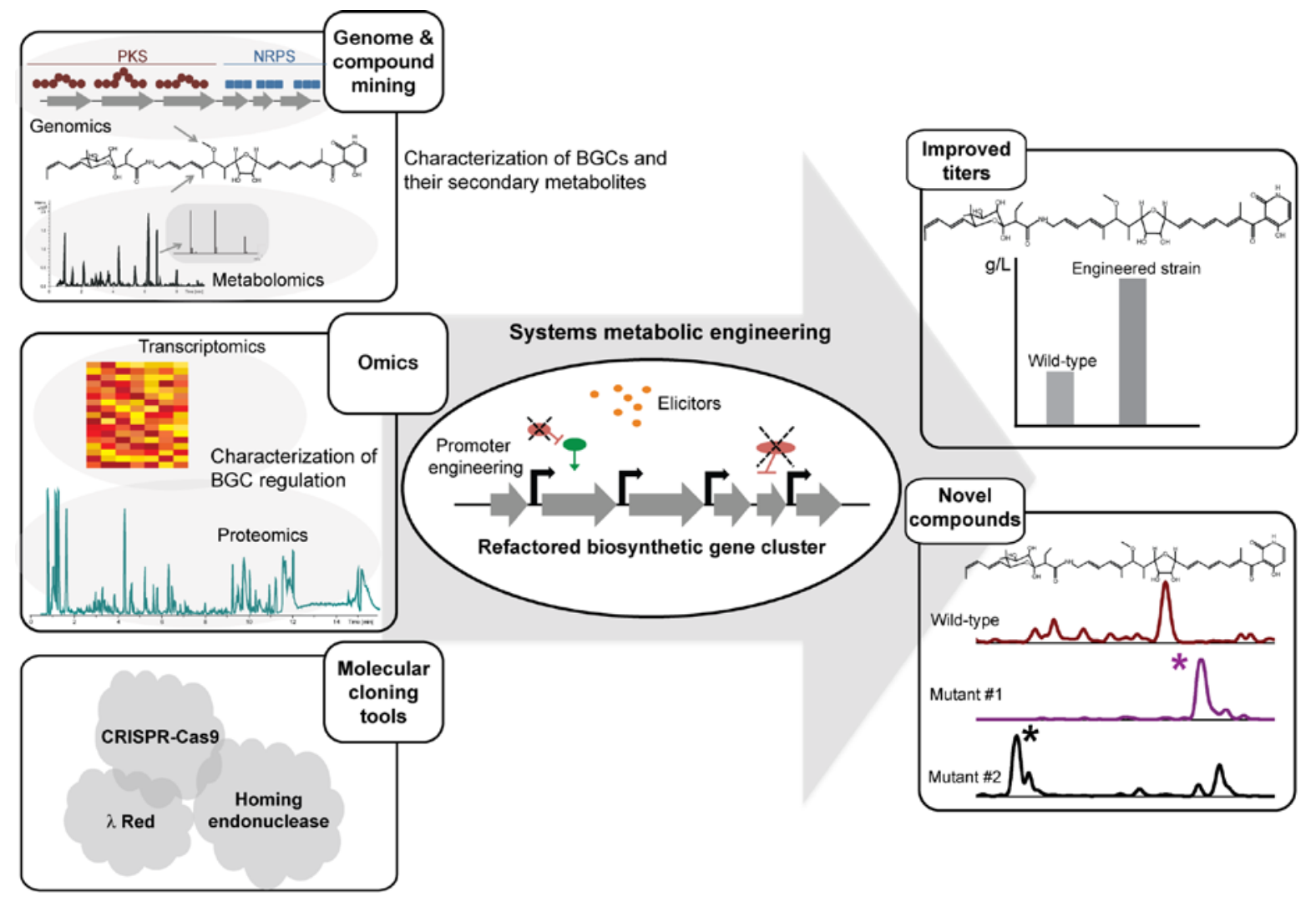

Figure. 2 


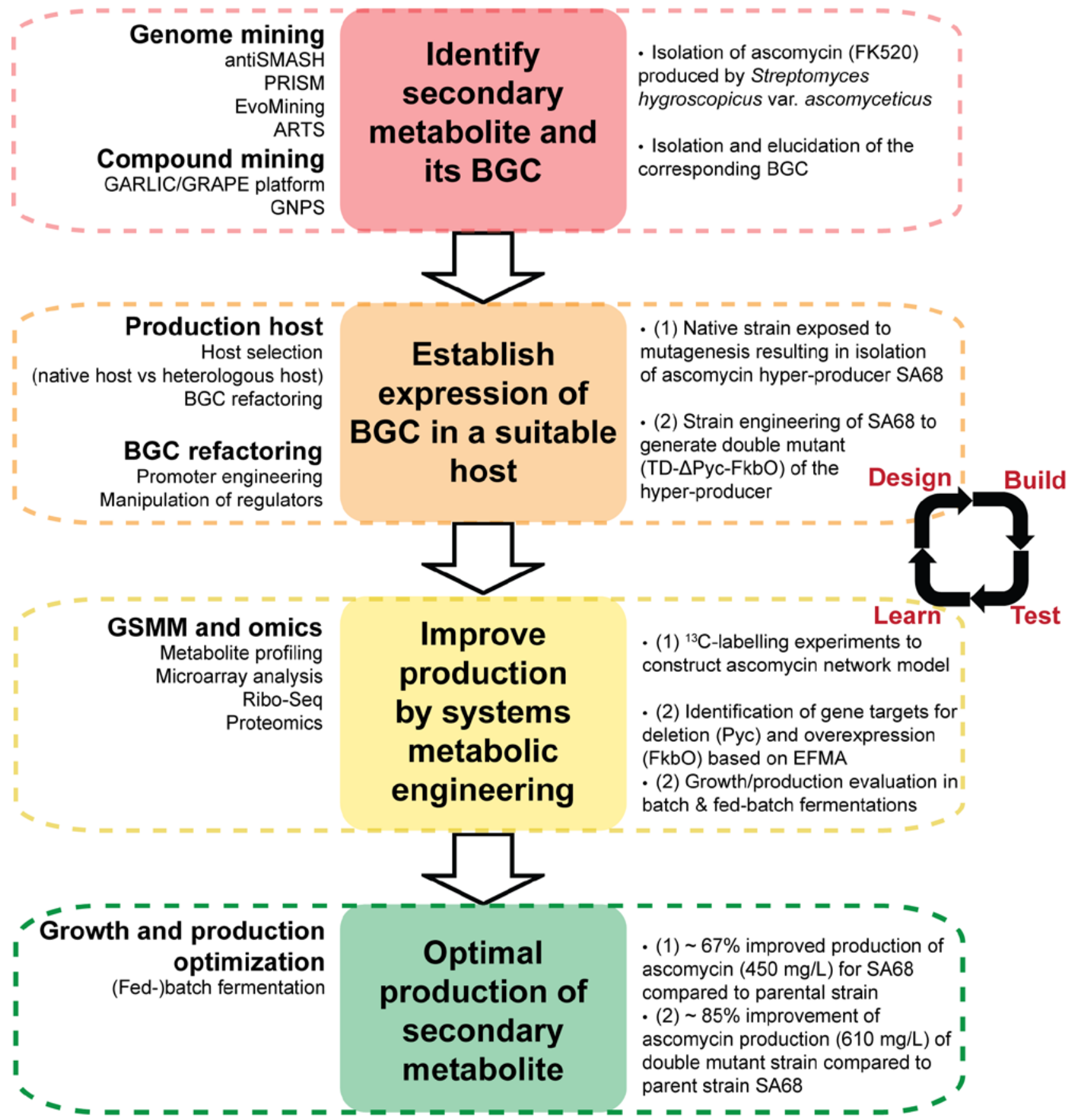

Figure. 3 